\title{
Arterial function of carotid and brachial arteries in postmenopausal vegetarians
}

This article was published in the following Dove Press journal:

Vascular Health and Risk Management

22 August 201I

Number of times this article has been viewed

\author{
Ta-Chen Su' \\ Pao-Ling Torng ${ }^{2}$ \\ Jiann-Shing Jeng ${ }^{3}$ \\ Ming-Fong Chen' \\ Chiau-Suong Liau ${ }^{1,4}$ \\ 'Division of Cardiology, \\ Department of Internal Medicine, \\ ${ }^{2}$ Department of Obstetrics and \\ Gynecology, ${ }^{3}$ Department of \\ Neurology, National Taiwan University \\ Hospital, National Taiwan University \\ College of Medicine, ${ }^{4} \mathrm{Cardiovascular}$ \\ Center, Taipei Buddist Tzu-Chi \\ Hospital, Hsin-Dian, Taipei, Taiwan
}

Correspondence: Ta-Chen Su Department of Internal Medicine, National Taiwan University Hospital and National Taiwan University College of Medicine, 7 Chung-Shan S Road, Taipei, Taiwan 10002

$\mathrm{Tel}+886223123456$ ext 66719

Fax +8862 237| 236।

Email tachensu@ntu.edu.tw
Background: Vegetarianism is associated with a lower risk of cardiovascular disease. However, studies of arterial function in vegetarians are limited.

Methods: This study investigated arterial function in vegetarianism by comparing 49 healthy postmenopausal vegetarians with 41 age-matched omnivores. The arterial function of the common carotid artery was assessed by carotid duplex, while the pulse dynamics method was used to measure brachial artery distensibility (BAD), compliance (BAC), and resistance (BAR). Fasting blood levels of glucose, lipids, lipoprotein (a), high-sensitivity C-reactive protein, homocysteine, and vitamin B12 were also measured.

Results: Vegetarians had significantly lower serum cholesterol, high-density and low-density lipoprotein, and glucose compared with omnivores. They also had lower vitamin B12 but higher homocysteine levels. Serum levels of lipoprotein (a) and high-sensitivity C-reactive protein were no different between the two groups. There were no significant differences in carotid beta stiffness index, BAC, and BAD between the two groups even after adjustment for associated covariates. However, BAR was significantly lower in vegetarians than in omnivores. Multiple linear regression analysis revealed that age and pulse pressure were two important determinants of carotid beta stiffness index and BAD. Vegetarianism is not associated with better arterial elasticity.

Conclusion: Apparently healthy postmenopausal vegetarians are not significantly better in terms of carotid beta stiffness index, BAC, and BAD, but have significantly decreased BAR than omnivores. Prevention of vitamin B12 deficiency might be beneficial for cardiovascular health in vegetarians.

Keywords: postmenopausal women, vegetarians, carotid stiffness, brachial arterial distensibility

\section{Introduction}

In vegetarians, better plasma lipid and lipoprotein profiles have been implicated in lower rates of cardiovascular disease.$^{1-4}$ However, the cardiovascular effects of a vegetarian diet are not well studied. Studies have demonstrated that vegetarians have lower levels of low-density and high-density lipoprotein cholesterol. ${ }^{3,4}$ Lin et al report that ovolactovegetarians have better endothelial function. ${ }^{5}$ However, vegetarians are observed to have vitamin B12 deficiency and elevated plasma homocysteine levels, ${ }^{3,6,7}$ which may attenuate the beneficial cardiovascular effects of vegetarian diets. ${ }^{3}$

A previous study has reported that there was no significant difference in carotid atherosclerosis between apparently healthy postmenopausal vegetarians and omnivores. ${ }^{3}$ Three major epidemiologic studies conducted in the United Kingdom all show no significant reduction in cardiovascular mortality in vegetarians compared with 
omnivores with similar lifestyles. ${ }^{8}$ Contrary to traditional belief, Kwok et al report that vegetarians may have abnormal arterial endothelial function and accelerated atherosclerosis compared with omnivores. ${ }^{9}$ Deterioration of elastic arterial structures and development of arterial stiffness is one of the possible underlying mechanisms. Thus, investigating the vascular characteristics of vegetarians in terms of arterial stiffness functions may help clarify the paradox of vegetarianism.

Arterial elasticity is considered to be a new cardiovascular risk factor ${ }^{10}$ and is documented to correlate highly with pathologic changes in the common carotid artery. ${ }^{11}$ Arterial elasticity is a general term that encompasses a variety of vascular properties, including distensibility, compliance, and elastic modulus. It is a result of natural aging and can be produced by many disease states, including diabetes, atherosclerosis, and chronic renal disease. In nondiabetic subjects, increased arterial stiffness has been implicated as an important risk factor for cardiovascular disease. ${ }^{12,13}$

This study aimed to compare plasma vitamin B12 and homocysteine levels as risk factors for cardiovascular disease, as well as measurements of carotid arterial beta stiffness indices and brachial arterial function variables between healthy postmenopausal vegetarians and matched omnivores.

\section{Materials and methods}

\section{Subjects}

Vegetarians were defined as subjects who were on diets that excluded meat, fish, and poultry for at least 5 years. Menopausal status was obtained from self-assessment questionnaire and only women who had undergone menopause at least 1 year previously were included.

Women with the following conditions were excluded: history of diabetes mellitus or fasting blood sugar $\geq 126 \mathrm{mg} / \mathrm{dL}$; significant hyperlipidemia possibly attributable to genetic variance, defined as serum cholesterol level $\geq 260 \mathrm{mg} / \mathrm{dL}$ or triglyceride levels $\geq 250 \mathrm{mg} / \mathrm{dL}$; lifestyle of regular alcohol drinking or tobacco use; history of cardiovascular disease; hypertension; serum creatinine level $\geq 125 \mu \mathrm{mol} / \mathrm{L}$; and malignancy. Subjects taking vitamin B12 pills were also excluded. ${ }^{3}$

A total of 49 postmenopausal vegetarians and 41 agematched and gender-matched omnivores recruited from volunteers for public service at the Buddhist Compassion ReliefTzu Chi Foundation in Taipei during 1999-2000, were included in this study. ${ }^{3}$ None of them had received hormonal replacement therapy in the previous 3 years. Written informed consent was obtained from each participant and the study was approved by the ethics committee of the institutional review board at National Taiwan University Hospital.

\section{Clinical and biochemical assays}

Blood samples were drawn from the antecubital vein while the patients were in a seated position after overnight fasting for 10-14 hours. Samples were stored at $4{ }^{\circ} \mathrm{C}$ and assayed for lipid profiles, including total cholesterol, high-density lipoprotein cholesterol, and triglycerides within 24 hours in the central laboratory of the National Taiwan University Hospital. Total cholesterol and triglyceride levels were measured by enzymatic methods using an automatic multichannel chemical analyzer (Hitachi 7450, Hitachi Corporation, Tokyo, Japan), while high-density lipoprotein cholesterol was determined by measuring cholesterol in the supernatant after precipitating very low-density lipoprotein cholesterol and low-density lipoprotein cholesterol with $\mathrm{Mg}^{2+}$ /phosphotungstate reagent. The low-density lipoprotein cholesterol level was calculated by Friedewald's formula.

For vitamin B12, folate, vitamin B6, homocysteine, lipoprotein (a), and high-sensitivity C-reactive protein measurements, blood samples were centrifuged at $3000 \mathrm{rpm}$ for 15 minutes within 60 minutes of collection and stored at $-70^{\circ} \mathrm{C}$ until analysis. Plasma levels of vitamin B12 and folate were measured by microparticle enzyme immunoassay technology using AxSYM ${ }^{\circledR}$ B12 and folate kits (Abbott Laboratories, Abbott Park, IL). Plasma vitamin B6 concentration was determined by high-pressure liquid chromatography and fluorometric detection (Hitachi, Tokyo, Japan), while plasma homocysteine was determined by competitive immunoassay with an Immulite ${ }^{\circledR}$ homocysteine kit (Diagnostic Products Corporation, Los Angeles, CA). A rate nephelometry method (Beckman Array ${ }^{\circledR}$ Systems, Indianapolis, IN) and two-site chemiluminescent enzyme immunometric assay (Immulite ${ }^{\circledR}$ C-reactive protein kit, Diagnostic Products Corporation) were used to determine lipoprotein (a) and serum high-sensitivity C-reactive protein, respectively.

\section{Measurements of carotid arterial elasticity}

The carotid artery luminal diameter at the common carotid artery and at $2 \mathrm{~cm}$ proximal to the common carotid artery bifurcation were measured in both the systolic and diastolic phases of the cardiac cycle using a high-resolution B-mode ultrasonographic system (Hewlett Packard SONO 4500 ultrasound system, Andover, MA) equipped with a $3-11 \mathrm{mHz}$ real-time B-mode scanner and a $3.6 \mathrm{mHz}$ pulsed-Doppler mode scanner. An experienced neurologist performed carotid 
ultrasonography, with the patient in a supine position and the neck extended in slight lateral rotation. All of the scans were recorded by a digital recording system for subsequent offline analysis.

In this study, the measurement protocol for carotid artery ultrasonography followed that of Gamble et al. ${ }^{14}$ In brief, the digitized M-mode ultrasonographic records were analyzed offline using a computer program in which the images were recalled and magnified. For each image, measurements were performed during one cardiac cycle, which was divided into 10 equal sections. The distance between the lumen-intima interfaces of the near and far walls of the carotid artery were measured by a computer-generated grid. ${ }^{15}$ Thus, the maximal (Ds) and the minimal carotid diameter (Dd) were obtained.

The elastic properties of the carotid artery wall, in terms of carotid beta stiffness index, was calculated from the carotid artery diameter and blood pressure of the brachial artery as follows: $\beta=\ln (\mathrm{Ps} / \mathrm{Pd}) \mathrm{Dd} /(\mathrm{Ds}-\mathrm{Dd})$. The Ps and Pd were the systolic and diastolic blood pressures of the brachial artery, respectively, Ds and Dd were the systolic and diastolic inner diameters of the carotid artery, respectively, and $\beta$ was the carotid beta stiffness index. ${ }^{14}$

\section{Brachial artery compliance and distensibility}

The arterial pressure waveform was recorded using a cuff sphygmomanometer with an oscillometric blood pressure device (DynaPulse 2000A, Pulse Metric Inc, San Diego, CA). ${ }^{16-19}$ Data was transferred online from the data collection site to a central analysis center. Blood pressure was determined by pressure waveform changes according to Bernoulli flow effects. Vascular compliance and peripheral resistance of the brachial artery were derived by incorporating the arterial pressure signals from a standard cuff sphygmomanometer using a physical model. This method was previously validated against invasive ${ }^{16}$ and noninvasive measurements. ${ }^{17}$

Brachial artery compliance (BAC) was calculated at mean arterial pressure by theoretical design. ${ }^{18}$ Brachial artery distensibility (BAD) and resistance (BAR) were derived from the modified formula of BAC, which was associated with cardiovascular risk factors in healthy young adults in the Bogalusa Heart Study. ${ }^{18,19}$

\section{Statistical analysis}

Baseline characteristics and all measurements were compared between vegetarians and omnivores by $t$-test and analysis of variance. Continuous variables were expressed as the mean \pm standard deviation. Lipoprotein (a) concentration was not in normal distribution and was analyzed using the Wilcoxon-Mann-Whitney test. For categorical data, the Chi-square test was used to test the significance level between different groups. Determinants of the carotid beta stiffness index and the distensibility coefficient were estimated by univariate and multivariate linear regression analyses after adjustment for associated covariates. Because of the issue of collinearity with age, "years after menopause" was not included as an item in the multivariate model. Measurements of arterial function obtained by pulse dynamic methods were compared between vegetarians and omnivores before and after adjusting for covariates.

\section{Results}

The cardiovascular characteristics of the 49 vegetarians are shown on Table 1. Their mean duration of adoption of a vegetarian diet was $10.2 \pm 6.7$ years. Most adopted a vegan type of vegetarian diet (consuming no eggs or milk) and only six were ovolactovegetarians. The age of menopause was 2.1 years earlier in the vegetarians and time after menopause was 3.3 years longer than the omnivores. Body mass index was the same for the two groups.

Vegetarians had significantly lower fasting serum glucose, total cholesterol, and low-density lipoprotein levels. Highdensity lipoprotein concentration was significantly lower in vegetarians than in omnivores. The plasma vitamin B12 concentration was lower and homocysteine was higher in vegetarians. There was a trend of lower lipoprotein (a) level in vegetarians, but this did not reach statistical significance.

As shown in Table 2, arterial elasticity, as expressed by the carotid beta stiffness index and BAD, was not significantly different between the two groups. After adjusting the associated risk factors, including age, levels of low-density lipoprotein, fasting glucose, lipoprotein (a), and pulse pressure, there were still no significant differences between the two groups. However, BAR was significantly lower in vegetarians than in omnivores, even after adjusting for the associated risk factors.

In Table 3, arterial elasticity as measured by the elastic property of the carotid arterial wall beta stiffness index, showed that age, duration of menopause, systolic blood pressure, and pulse pressure were significant determinants of carotid stiffness. Significant determinants of BAD included fasting glucose, age, systolic blood pressure, and pulse pressure (Table 3 ). In multivariate analysis, age and pulse pressure were the major determinants for carotid beta stiffness index, while age, pulse pressure, and fasting blood glucose the major determinants of BAD (Table 4). 
Table I Basic characteristics of vegetarians and omnivores

\begin{tabular}{|c|c|c|c|}
\hline Characteristics & $\begin{array}{l}\text { Vegetarians } \\
n=49\end{array}$ & $\begin{array}{l}\text { Omnivores } \\
n=41\end{array}$ & $P$ value \\
\hline Age, years & $58.6 \pm 6.0$ & $57.2 \pm 5.4$ & 0.255 \\
\hline Vegetarian duration, years & $10.8 \pm 7.5$ & - & - \\
\hline Age at menopause, years & $47.2 \pm 4.8$ & $49.7 \pm 4.4$ & 0.013 \\
\hline Years after menopause & $11.6 \pm 6.3$ & $7.5 \pm 6.6$ & 0.004 \\
\hline Body mass index, $\mathrm{kg} / \mathrm{m}^{2}$ & $23.2 \pm 2.7$ & $23.1 \pm 2.9$ & 0.966 \\
\hline Systolic BP, mmHg & $128.8 \pm 14.2$ & $128.0 \pm 14.0$ & 0.800 \\
\hline Diastolic BP, mmHg & $70.8 \pm 7.1$ & $70.6 \pm 8.3$ & 0.893 \\
\hline Pulse pressure, $\mathrm{mmHg}$ & $58.0 \pm 11.0$ & $57.4 \pm 11.2$ & 0.817 \\
\hline Cholesterol, $\mathrm{mmol} / \mathrm{L}$ & $5.0 \pm 0.8$ & $5.5 \pm 0.7$ & 0.005 \\
\hline $\mathrm{HDL}-\mathrm{C}, \mathrm{mmol} / \mathrm{L}$ & $\mathrm{I} .5 \pm 0.3$ & $\mathrm{I} .7 \pm 0.4$ & 0.002 \\
\hline LDL-C, $\mathrm{mmol} / \mathrm{L}$ & $2.8 \pm 0.7$ & $3.2 \pm 0.6$ & 0.016 \\
\hline Fasting glucose, $\mathrm{mmol} / \mathrm{L}$ & $5.0 \pm 0.5$ & $5.2 \pm 0.5$ & 0.019 \\
\hline Folate, $\mathrm{nmol} / \mathrm{L}$ & $22.6 \pm 7.6$ & $22.8 \pm 5.9$ & 0.875 \\
\hline Vitamin BI2, pmol/L & $273.4 \pm 184.8$ & $359.7 \pm 138.1$ & 0.016 \\
\hline Vitamin B6, nmol/L & $167.5 \pm 87.1$ & $149.2 \pm 68.4$ & 0.383 \\
\hline Homocysteine, $\mathrm{mol} / \mathrm{L}$ & $10.8 \pm 3.2$ & $9.2 \pm 2.2$ & 0.005 \\
\hline Lipoprotein (a), mg/dL & $6.1 \pm 5.8$ & $7.0 \pm 5.7$ & 0.157 \\
\hline hs-CRP, mg/dL & $0.07 \pm 0.07$ & $0.09 \pm 0.12$ & 0.436 \\
\hline
\end{tabular}

Abbreviations: BP, blood pressure; HDL-C, high-density lipoprotein cholesterol; LDL-C, low-density lipoprotein cholesterol; hs-CRP, high-sensitivity C-reactive protein.

\section{Discussion}

Vegetarianism is not only a public health concern but is also a model of interaction between environmental factors and health. Despite the well recognized reduction in low-density lipoprotein and blood sugar, there is no difference in BAD and carotid stiffness and only a moderate decrease in BAR in apparently healthy postmenopausal vegetarians compared with matched omnivores. The better plasma lipid profiles of vegetarians implies a lower cardiovascular risk. Among the study subjects, age at menopause was younger and duration since menopause was longer for the vegetarians, which may contribute to changes in arterial function. In addition, lower high-density lipoprotein and elevated homocysteine may also attenuate the beneficial cardiovascular effects of lower low-density lipoprotein levels in vegetarians. Nevertheless, this study provides evidence of higher arterial elasticity in terms of decreased BAR in vegetarians.

The negative findings of this study should not be considered as evidence of neutral cardiovascular effects of vegetarianism, but do indicate an urgent need for modification of vegan diets through vitamin B12 fortification or supplements. Although recommendations for vitamin B12 are very small (recommended daily allowance $2.4 \mu \mathrm{g}$ daily for adults), vitamin B12 deficiency is a very serious problem leading ultimately to anemia, neuropsychiatric disorders, irreversible nerve damage, ${ }^{20}$ and relative hyperhomocysteinemia..$^{5,8,9,20}$ Prudent vegans should include sources of vitamin B12 in their diets.

Table 2 Comparisons of carotid and brachial arterial elasticity indices between vegetarians and omnivores

\begin{tabular}{|c|c|c|c|c|}
\hline & \multicolumn{2}{|l|}{ Model I } & \multicolumn{2}{|l|}{ Model 2} \\
\hline & Vegetarians & Omnivores & Vegetarians & Omnivores \\
\hline \multicolumn{5}{|l|}{ Carotid stiffness } \\
\hline Right & $9.81 \pm 7.17$ & $8.06 \pm 3.47$ & $9.73 \pm 0.85$ & $8.15 \pm 0.95$ \\
\hline Left & $9.39 \pm 4.46$ & $8.21 \pm 3.61$ & $9.24 \pm 0.53$ & $8.40 \pm 0.59$ \\
\hline Mean & $9.60 \pm 4.40$ & $8.14 \pm 2.93$ & $9.49 \pm 0.52$ & $8.28 \pm 0.58$ \\
\hline \multicolumn{5}{|c|}{ Brachial arterial elasticity index } \\
\hline $\mathrm{BAC}, \mathrm{mL} / \mathrm{mmHg}$ & $0.055 \pm 0.012$ & $0.051 \pm 0.011$ & $0.054 \pm 0.002$ & $0.051 \pm 0.002$ \\
\hline $\mathrm{BAD}, \% / \mathrm{mmHg}$ & $5.66 \pm 1.13$ & $5.73 \pm 1.4 \mid$ & $5.72 \pm 0.18$ & $5.65 \pm 0.20$ \\
\hline $\mathrm{BAR}, \mathrm{mmHg} / \mathrm{L} / \mathrm{min}$ & $4122 \pm 1418$ & $4977 \pm 1936^{*}$ & $4184 \pm 243$ & $4904 \pm 266^{*}$ \\
\hline
\end{tabular}

Note: Data are expressed as the least squares mean \pm standard error of least squares mean; $* P<0.05$.

Abbreviations: BAC, brachial artery compliance; BAD, brachial artery distensibility; BAR, brachial artery resistance; Model I, nonadjusted data; Model 2, meansadjusted for age. 
Table 3 Univariate analysis for determinants of carotid and brachial arterial elasticity indices

\begin{tabular}{lcc}
\hline Characteristics & $\begin{array}{c}\text { Carotid stiffness, } \beta \\
\text { parameter estimate (SE) }\end{array}$ & $\begin{array}{c}\text { Brachial artery distensibility } \\
\text { parameter estimate (SE) }\end{array}$ \\
\hline Age, years & $0.26 \pm 0.07^{\ddagger}$ & $-0.06 \pm 0.02^{*}$ \\
Years after menopause & $0.20 \pm 0.06^{\dagger}$ & $-0.03 \pm 0.02$ \\
Systolic BP, $\mathrm{mmHg}$ & $0.08 \pm 0.03^{\dagger}$ & $-0.05 \pm 0.01^{\ddagger}$ \\
Diastolic BP, $\mathrm{mmHg}$ & $-0.08 \pm 0.05$ & $-0.03 \pm 0.02$ \\
Pulse pressure, $\mathrm{mmHg}$ & $0.16 \pm 0.03^{\ddagger}$ & $-0.06 \pm 0.01^{\ddagger}$ \\
Vegetarianism & $1.46 \pm 0.82$ & $-0.06 \pm 0.27$ \\
Homocysteine, $\mathrm{mol} / \mathrm{L}$ & $-0.01 \pm 0.15$ & $-0.02 \pm 0.05$ \\
Fasting glucose, $\mathrm{mmol} / \mathrm{L}$ & $-0.09 \pm 0.85$ & $-0.65 \pm 0.27^{*}$ \\
LDL-C, mmol/L & $-0.08 \pm 0.62$ & $0.16 \pm 0.20$ \\
HDL-C, mmol/L & $-0.83 \pm 1.13$ & $0.09 \pm 0.37$ \\
Body mass index, $\mathrm{kg} / \mathrm{m}^{2}$ & $0.15 \pm 0.15$ & $-0.04 \pm 0.05$ \\
hs-CRP, mg/dl & $-3.60 \pm 6.03$ & $-0.37 \pm 1.74$ \\
\hline
\end{tabular}

Notes: $* P<0.05,+P<0.01, \ddagger P<0.005$.

Abbreviations: BP, blood pressure; HDL-C, high-density lipoprotein cholesterol; LDL-C, low-density lipoprotein cholesterol; hs-CRP, high-sensitivity C-reactive protein; SE, standard error.

Plasma homocysteine has emerged as a risk factor for cardiovascular disease, ${ }^{21}$ and even mildly elevated homocysteine can cause an exacerbation of neointima formation in rat carotid arteries after endothelial injury. ${ }^{22}$ Mild hyperhomocysteinemia is also associated with metalloproteinase-related elastolysis and subsequent structural deterioration in the minipig arteries, which may be one of the major events underlying the epidemiological association between mild hyperhomocysteinemia and arterial disease. ${ }^{23} \mathrm{~A}$ methioninerich diet induces hyperhomocysteinemia with associated diffuse arterial lesions, smooth muscle cell hyperplasia, endothelial alterations, and elastic lamina dislocation. ${ }^{24}$ This study, as well as previous studies, ${ }^{3,6,7}$ demonstrates that vegetarians have lower vitamin B12 and elevated plasma homocysteine, which may attenuate the favorable lipid effects of vegetarian diets on arterial stiffness. Hence, the mild hyperhomocysteinemia in vegetarians may partly be the reason for similar, and not better, arterial compliance than in omnivores. However, this study shows a significant decrease in BAR in vegetarians, indicating a potential beneficial effect of vegetarianism. One study has reported that BAR is a significant risk factor for coronary artery calcification measured by electron beam tomography in women, ${ }^{25}$ This may support the potential beneficial effect of vegetarianism in this study.

Vegetarians in Taiwan take many soybean products. ${ }^{6}$ Soybean is known to be rich in phytoestrogens, which have been associated with favorable metabolic effects on the cardiovascular system in postmenopausal women. ${ }^{26} \mathrm{~A}$ higher phytoestrogen intake is also associated with lower arterial stiffness in postmenopausal women. ${ }^{27}$ Consistent with a previous Australian study, ${ }^{28}$ our study shows a trend of lower lipoprotein (a) levels in vegetarians. It also shows that high-sensitivity $\mathrm{C}$-reactive protein is not different between vegetarians and omnivores, and reveals no association between high-sensitivity C-reactive protein and arterial elasticity.

Table 4 Multiple linear regression analysis for the determinants of carotid and brachial arterial elasticity indices

\begin{tabular}{|c|c|c|}
\hline Characteristics & $\begin{array}{l}\text { Carotid stiffness, } \beta \\
\text { parameter estimate (SE) }\end{array}$ & $\begin{array}{l}\text { Brachial artery distensibility } \\
\text { parameter estimate (SE) }\end{array}$ \\
\hline Intercept & $-6.26 \pm 5.46$ & $11.29 \pm 1.75^{\ddagger}$ \\
\hline Age, years & $0.15 \pm 0.07 *$ & $0.05 \pm 0.02 *$ \\
\hline Pulse pressure, $\mathrm{mmHg}$ & $0.14 \pm 0.04^{\ddagger}$ & $-0.06 \pm 0.0 \mathrm{I}^{\ddagger}$ \\
\hline Vegetarianism & $1.15 \pm 0.80$ & $-0.02 \pm 0.26$ \\
\hline Fasting glucose, $\mathrm{mmol} / \mathrm{L}$ & $-0.57 \pm 0.78$ & $-0.62 \pm 0.25^{*}$ \\
\hline LDL-C, mmol/L & $0.54 \pm 0.58$ & $0.10 \pm 0.18$ \\
\hline HDL-C, mmol/L & $-0.53 \pm 1.06$ & $0.27 \pm 0.34$ \\
\hline Adjusted $\mathrm{R}^{2}$ & 0.2494 & 0.2803 \\
\hline
\end{tabular}

Notes: $* P<0.05,+p<0.01, \stackrel{\ddagger}{\dagger}<0.005$.

Abbreviations: HDL-C, high-density lipoprotein cholesterol; LDL-C, low-density lipoprotein cholesterol; SE, standard error. 
Serum glucose is lower for vegetarians, as previously demonstrated in these study subjects. ${ }^{3}$ It has been reported that vegetarians have higher insulin sensitivity than omnivores. ${ }^{29}$ Aside from age and pulse pressure, this study also highlights blood glucose as being a significant determinant of BAD. The finding of higher fasting glucose associated with decreased arterial elasticity has been previously reported..$^{30,31}$ Thus, the favorable aspects of lower levels of blood cholesterol and glucose, and a trend of lower lipoprotein (a) levels in vegetarians, deserve more attention.

In this study, pulse pressure was a major determinant of arterial elasticity, which is compatible with our previous population-based study in which pulse pressure was associated with carotid atherosclerosis, independent of hypertension status. ${ }^{32}$ Diabetes mellitus is also notably an important determinant of pulse pressure. ${ }^{32}$ Our previous study in nondiabetic middle-aged subjects demonstrated that postchallenge hyperglycemia and systolic blood pressure are two major determinants of aortic stiffness. ${ }^{33}$ Thus, increased pulse pressure and hyperglycemia have significant adverse effects on both arterial elasticity and atherosclerosis.

Recently, several noninvasive tools have been developed for evaluating early vascular changes and for application in clinical practice. Noninvasive pulse wave analysis has been applied to estimate arterial stiffness and the association with vascular diseases. ${ }^{15,34}$ Arterial elasticity, in terms of vascular distensibility and compliance, can be measured by waveform analysis of arterial pressure signals using a standard cuff sphygmomanometer at the brachial artery. ${ }^{16-19}$ In the present study, carotid beta stiffness index and BAD were applied as arterial elasticity indices that might be more sensitive than carotid atherosclerosis for the study of early vascular changes in these relatively healthy subjects. Surprisingly, the results show no difference in arterial elasticity indices between vegetarians and omnivores.

By multivariate linear regression analysis, pulse pressure is also a major determinant for carotid beta stiffness index and BAD. Age and years after menopause also positively correlate with carotid beta stiffness index. Early vascular changes, such as reduction in arterial compliance and distensibility, can be detected before clinical manifestation of systolic blood pressure elevation, pulse pressure widening, and left ventricular hypertrophy, ${ }^{34}$ which are processes of coronary heart disease and hypertensive heart disease. ${ }^{35}$

There are some limitations to this study. There is a possibility that the sample size is not sufficient to rule out a type 2 error and that there may be a real difference between the groups but the sample size was too small to identify it.
Second, the cross-sectional design cannot infer a causal relationship. Third, the relatively young ages of the study subjects may have limited the development of atherosclerosis. It is possible that with a longer follow-up period, more arterial changes may have been detected. Nonetheless, this study incorporated two kinds of arterial elasticity indices, i.e. carotid beta stiffness index and brachial arterial function of BAD, to demonstrate the null hypothesis of cardiovascular effects of vegetarianism, which may underscore the importance of micronutrients in the health of such a specific population.

\section{Conclusion}

There is no significant difference in carotid beta stiffness index and brachial arterial elasticity in terms of BAC and BAD between apparently healthy postmenopausal vegetarians and omnivores. However, BAR is significantly lower in vegetarians. Postmenopausal vegetarian women have lower plasma vitamin B12 but higher homocysteine levels, and earlier menopause, which may attenuate the beneficial effects of a vegetarian diet on cardiovascular health. The beneficial effects of vegetarian diets on lipids and blood glucose need to be advocated, and efforts to correct vitamin B12 deficiency in vegetarian diets can never be overestimated.

\section{Acknowledgments}

This study was supported jointly by grants from the National Taiwan University Hospital and the National Health Research Institute (NHRI) under grant \#NHRI-EX97-9721PC.

\section{Disclosure}

The authors report no conflicts of interest in this work.

\section{References}

1. Thorogood M. Vegetarianism, coronary disease risk factors and coronary heart disease. Curr Opin Lipidol. 1994;1:17-21.

2. Sacks FM, Castelli WP, Donner A, Kass EH. Plasma lipids and lipoproteins in vegetarians and controls. N Engl J Med. 1975;292: 1148-1151.

3. Su TC, Jeng JS, Wang JD, et al. Homocysteine, circulating vascular cell adhesion molecule and carotid atherosclerosis in post-menopausal vegetarian women and omnivores. Atherosclerosis. 2006;184:356-362.

4. Pan WH, Chin CJ, Sheu CT, Lee MH. Hemostatic factors and blood lipids in young Buddhist vegetarians and omnivores. Am J Clin Nutr. 1993;58:354-359.

5. Lin CL, Fang TC, Gueng MK. Vascular dilatory functions of ovolactovegetarians compared with omnivores. Atherosclerosis. 2001;158: 247-251.

6. Hung CJ, Huang PJ, Lu SC, et al. Plasma homocysteine levels in Taiwanese vegetarians are higher than those of omnivores. J Nutr. 2002;132: $152-158$.

7. Herrmann W, Schorr H, Purschwitz K, Rassoul F, Richter V. Total homocysteine, vitamin B12, and total antioxidant status in vegetarians. Clin Chem. 2001;47:1094-1101. 
8. Key TJ, Appleby PN, Spencer EA, Travis RC, Roddam AW, Allen NE. Mortality in British vegetarians: results from the European Prospective Investigation into Cancer and Nutrition (EPIC-Oxford). Am J Clin Nutr. 2009;89:1613S-1619S.

9. Kwok T, Chook P, Tam L, et al. Vascular dysfunction in Chinese vegetarians: an apparent paradox? J Am Coll Cardiol. 2005;46:1957-1958.

10. Arnett DK, Evans GW, Riley WA. Arterial stiffness: a new cardiovascular risk factor? Am J Epidemiol. 1994;140:669-682.

11. Wada T, Kodaira K, Fujishiro K, et al. Correlation of ultrasoundmeasured common carotid artery stiffness with pathological findings. Arterioscler Thromb. 1994;14:479-482.

12. Westerhof N, O'Rouke MF. Hemodynamic basis for the development of left ventricular failure in systolic hypertension and for its logical therapy. J Hypertens. 1995;13:943-952.

13. Sutton-Tyrrell K, Najjar SS, Boudreau RM, et al. Elevated aortic pulse wave velocity, a marker of arterial stiffness, predicts cardiovascular events in well-functioning older adults. Circulation. 2005; 111:3384-3390.

14. Gamble G, Zorn J, Sanders G, MacMahon S, Sharpe N. Estimation of arterial stiffness, compliance, and distensibility from M-mode ultrasound measurements of the common carotid artery. Stroke. 1994; 25:11-16.

15. Reneman RS, van Merode T, Hick P, Hoeks APG. Cardiovascular applications of multi-gate pulsed Doppler systems. Ultrasound Med Biol. 1986;12:357-370.

16. Brinton TJ, Cotter B, Kailasam MT, et al. Development and validation of a non-invasive method to determine arterial pressure and vascular compliance. Am J Cardiol. 1997;80:323-330.

17. Brinton TJ, Daniel Walls E, Chio SS. Validation of pulse dynamic blood pressure measurement by auscultation. Blood Press Monit. 1998;3: $121-124$.

18. Urbina EM, Kieltkya L, Tsai J, Srinivasan SR, Berenson GS. Impact of multiple cardiovascular risk factors on brachial artery distensibility in young adults: The Bogalusa Heart Study. Am J Hypertens. 2005; 18:767-771.

19. Urbina EM, Brinton TJ, Elkasabany A, Berenson GS. Brachial artery distensibility and relation to cardiovascular risk factors in healthy young adults (The Bogalusa Heart Study). Am J Cardiol. 2002;89:946-951.

20. Craig WJ. Health effects of vegan diets. Am J Clin Nutr. 2009;89:1627S-1633S.

21. Clarke R, Daly L, Robinson K, et al. Hyperhomocysteine: An independent risk factor for vascular disease. $N$ Engl J Med. 1991;324:149-155.
22. Morita H, Kurihara H, Yoshida S, et al. Diet-induced hyperhomocysteinemia exacerbates neo-intima formation in rat carotid arteries after balloon injury. Circulation. 2001;103:133-139.

23. Charpiot P, Bescond A, Augier T, et al. Hyperhomocysteinemia induces elastolysis in minipig arteries: structural consequences, arterial site specificity and effect of captopril-hydrochlorothiazide. Matrix Biol. 1998; 17:559-574.

24. Ambrosi P, Rolland PH, Bodard H, et al. Effects of folate supplementation in hyperhomocysteinemic pigs. J Am Coll Cardiol. 1999;34: 274-279.

25. Budoff MJ, Flores F, Tsai J, et al. Measures of brachial artery distensibility in relation to coronary calcification. Am J Hypertens. 2003; 16(5 Pt 1):350-355.

26. de Kleijn MJ, van der Schouw YT, Wilson PW, Grobbee DE, Jacques PF. Dietary intake of phytoestrogens is associated with a favorable metabolic cardiovascular risk profile in post-menopausal U.S. women: the Framingham study. J Nutr. 2002;132:276-282.

27. van der Schouw YT, Pijpe A, Lebrun CE, et al. Higher usual dietary intake of phytoestrogens is associated with lower aortic stiffness in post-menopausal women. Arterioscler Thromb Vasc Biol. 2002;22:1316-1322.

28. Li D, Ball M, Bartlett M, Sinclair A. Lipoprotein(a), essential fatty acid status and lipoprotein lipids in female Australian vegetarians. Clin Sci (Lond). 1999;97:175-181.

29. Hung CJ, Huang PC, Li YH, et al. Taiwanese vegetarians have higher insulin sensitivity than omnivores. Br J Nutr. 2006;95:129-135.

30. Henry RM, Kostense PJ, Spijkerman AM, et al. Arterial stiffness increases with deteriorating glucose tolerance status: the Hoorn study. Circulation. 2003;107:2089-2095.

31. Sengstock DM, Vaitkevicius PV, Supiano MA. Arterial stiffness is related to insulin resistance in nondiabetic hypertensive older adults. J Clin Endocrinol Metab. 2005;90:2823-2827.

32. Su TC, Chien KL, Jeng JS, et al. Pulse pressure, aortic regurgitation and carotid atherosclerosis: a comparison between hypertensives and normotensives. Int J Clin Pract. 2006;60:134-140.

33. Huang CL, Chen MF, Jeng JS, et al. Post-challenge hyperglycemic spike associated with arterial stiffness. Int J Clin Pract. 2007;61:397-402.

34. Roman MJ, Ganav A, Saba PS, et al. Impact of arterial stiffening on left ventricular structure. Hypertension. 2000;36:489-494.

35. Safar ME, Blancher J, Mourad JJ, London GM. Stiffness of carotid artery wall material and blood pressure in humans: application to antihypertensive therapy and stroke prevention. Stroke. 2000;31:782-790.
Vascular Health and Risk Management

\section{Publish your work in this journal}

Vascular Health and Risk Management is an international, peerreviewed journal of therapeutics and risk management, focusing on concise rapid reporting of clinical studies on the processes involved in the maintenance of vascular health; the monitoring, prevention and treatment of vascular disease and its sequelae; and the involvement of

\section{Dovepress}

metabolic disorders, particularly diabetes. This journal is indexed on PubMed Central and MedLine. The manuscript management system is completely online and includes a very quick and fair peer-review system, which is all easy to use. Visit http://www.dovepress.com/ testimonials.php to read real quotes from published authors. 